\title{
The Role of Deferiprone in Iron Chelation
}

TO THE EDITOR: In their review article, Hider and Hoffbrand (Nov. 29 issue) ${ }^{1}$ focus on iron chelation in patients with neurodegenerative diseases. However, we were disappointed that there was no mention of classic superficial siderosis of the central nervous system, one of the few progressive neurologic conditions for which iron chelation with deferiprone is currently used as a treatment. ${ }^{2,3}$

Classic superficial siderosis is characterized by hemosiderin deposits (typically in the superior cerebellar vermis, brain stem, and spinal cord $)^{2}$ that are easily identified on blood-sensitive magnetic resonance imaging (MRI) sequences. This condition is usually associated with the classic clinical triad of sensorineural hearing loss, ataxia, and myelopathy. The usual underlying cause of classic superficial siderosis is chronic low-volume subarachnoid hemorrhage, ${ }^{4}$ which is often associated with a traumatic or postsurgical dural de- fect. Although radiologic or surgical treatment of the source of bleeding is preferred, in patients in whom no such "leak" can be identified or repaired (or in those who cannot receive invasive treatment), iron chelation is an alternative or adjunctive option. ${ }^{2}$

Initial observational data on the use of deferiprone in patients with superficial siderosis are promising. ${ }^{3}$ However, data from randomized, controlled trials on efficacy and toxic effects (including agranulocytosis) ${ }^{5}$ are lacking.

\section{Gargi Banerjee, M.R.C.P.}

Yezen Sammaraiee, M.B., B.S.

David J. Werring, Ph.D., F.R.C.P.

University College London Queen Square Institute of Neurology London, United Kingdom

d.werring@ucl.ac.uk

No potential conflict of interest relevant to this letter was reported. 
1. Hider RC, Hoffbrand AV. The role of deferiprone in iron chelation. N Engl J Med 2018;379:2140-50.

2. Wilson D, Chatterjee F, Farmer SF, et al. Infratentorial superficial siderosis: classification, diagnostic criteria, and rational investigation pathway. Ann Neurol 2017;81:333-43.

3. Kessler RA, Li X, Schwartz K, Huang H, Mealy MA, Levy M. Two-year observational study of deferiprone in superficial siderosis. CNS Neurosci Ther 2018;24:187-92.

4. Fearnley JM, Stevens JM, Rudge P. Superficial siderosis of the central nervous system. Brain 1995;118:1051-66.

5. Huprikar N, Gossweiler M, Callaghan M, Bunge P. Agranulocytosis with deferiprone treatment of superficial siderosis. BMJ Case Rep 2013;2013:bcr2013010099.

DOI: 10.1056/NEJMc1817335 